\title{
On the domain of oddness of an infimal convolution
}

Tamás Glavosits and Csaba Kézi 


\title{
ON THE DOMAIN OF ODDNESS OF AN INFIMAL CONVOLUTION
}

\author{
TAMÁS GLAVOSITS AND CSABA KÉZI
}

Received 2 December, 2009

\begin{abstract}
To illustrate a recent uniqueness result on Hahn-Banach extensions, we show that if $p$ is a sublinear functional of a vector space $X, \varphi$ is a linear functional of a subspace $V$ of $X$ such that $\varphi(v) \leq p(v)$ for all $v \in V$, and

$$
q(x)=\inf _{v \in V}(p(x-v)+\varphi(v))
$$

for all $x \in X$, then the domain

$$
W=\{x \in X: \quad q(-x)=-q(x)\}
$$

of the oddness of the infimal convolution $q$ of $p$ and $\varphi$ is a subspace of $X$ such that any of the three possible cases $V=W, V \neq W \neq X$ and $W=X$ can naturally occur even if $X$ is an Euclidean space and $p$ is a norm on $X$.
\end{abstract}

2000 Mathematics Subject Classification: Primary 46A22; Secondary 26A99

Keywords: Hahn-Banach uniqueness, infimal convolution, domain of oddness

\section{INTRODUCTION}

Suppose that $X$ is a vector space over $\mathbb{R}$ and $p$ is a sublinear (positively homogeneous and subadditive) function on $X$ to $\mathbb{R}$.

Moreover, assume that $V$ is a subspace of $X$ and $\varphi$ is a linear function on $V$ to $\mathbb{R}$ such that $\varphi$ is dominated by $p$ on $V$ in the sense that $\varphi(v) \leq p(v)$ for all $v \in V$.

Now, because of the classical Hahn-Banach theorem [1,4], a linear function $f$ on $X$ to $\mathbb{R}$, that extends $\varphi$ and is dominated by $p$ on $X$, may be called a Hahn-Banach extension of $\varphi$ to $X$.

Moreover, in accordance with the more general definitions of Moreau [1,3], and Strömberg [5], the function $q=p * \varphi$, defined by

$$
q(x)=\inf _{v \in V}(p(x-v)+\varphi(v))
$$

for all $x \in X$, can be called the infimal convolution of $p$ and $\varphi$.

Under the above notations, the following theorem has been proved in [2] on the uniqueness of Hahn-Banach extension of $\varphi$ to $X$. 
Theorem 1. The following assertions are equivalent:

(1.) $q$ is odd on $X$;

(2.) $q$ is a Hahn-Banach extension of $\varphi$ to $X$;

(3.) there exists a unique Hahn-Banach extension $f$ of $\varphi$ to $X$;

(4.) there exists at most one Hahn-Banach extension $f$ of $\varphi$ to $X$.

This theorem has motivated us to investigate the domain

$$
W=\{x \in X: q(-x)=-q(x)\}
$$

of oddness of the function $q$.

By using some basic properties of $q$, we shall show that $W$ is always a subspace of $X$ containing $V$. Moreover, by using a particular infimal value theorem, we shall show that any of the three possible cases $V=W, V \neq W \neq X$, and $W=X$ can naturally occur even if $X$ is an Euclidean space and $p$ is a norm on $X$.

\section{LINEARITY PROPERTIES OF $q$ AND $W$}

The following two basic theorems have also been established in [2].

Theorem 2. $q$ is a real-valued, sublinear function of $X$.

Corollary 1. $q(0)=0$ and $\lambda q(x) \leq q(\lambda x)$ for all $\lambda \in \mathbb{R}$ and $x \in X$.

Remark 1 . Hence, one can easily derive that $q(\lambda x)=\lambda q(x)$ for all $\lambda \geq 0$ and $x \in X$.

Theorem 3. $q(x+v)=q(x)+\varphi(v)$ for all $x \in X$ and $v \in V$.

Corollary 2. $q$ is an extension of $\varphi$ and $q(x+v)=q(x)+q(v)$ for all $x \in X$ and $v \in V$.

Now, by using the above results, we can easily prove the following

Theorem 4. $W$ is a subspace of $X$ containing $V$.

Proof. Since $\varphi$ is odd and $q$ is an extension of $\varphi$, it is clear that $V \subset W$. Therefore, we only need to show that $W$ is closed under the linear operations in $X$.

For this, note first that if $x \in W$, then $q(-x)=-q(x)$. Hence, it is clear that

$$
q(-(-x))=q(x)=-(-q(x))=-q(-x) .
$$

Therefore, $-x \in W$ also holds.

Moreover, if $\lambda \geq 0$ and $x \in W$, then by Remark 2.3 and since $q(-x)=-q(x)$, we have

$$
q(-\lambda x)=q(\lambda(-x))=\lambda q(-x)=\lambda(-q(x))=-\lambda q(x)=-q(\lambda x) .
$$

Therefore, $\lambda x \in W$ also holds. 
While, if $\lambda<0$ and $x \in W$, then $-\lambda>0$ and $-x \in W$. Therefore, by the above observation, we also have

$$
\lambda x=(-\lambda)(-x) \in W .
$$

Finally, we note that if $x, y \in W$, then the subadditivity of $q$, the equalities $q(-x)=$ $-q(x)$ and $q(-y)=-q(y)$, and the $\lambda=-1$ particular case of Corollary 2.2 altogether imply

$$
\begin{aligned}
q(-(x+y))=q(-x+(-y)) & \leq q(-x)+q(-y) \\
=-q(x)+(-q(y)) & =-(q(x)+q(y)) \leq-q(x+y) \leq q(-(x+y))
\end{aligned}
$$

Therefore,

$$
q(-(x+y))=-q(x+y),
$$

and thus $x+y \in W$ also holds.

Remark 2. To appreciate the importance of $W$, note that by definition $W$ is the largest subset of $X$ such that $q$ is odd on $W$.

Hence, by Theorems 2.6 and 1.1, we can see that $W$ is the largest subspace of $X$ such that $\varphi$ has a unique Hahn-Banach extension $\psi$ to $W$. Moreover, we necessarily have $\psi(w)=q(w)$ for all $w \in W$.

\section{A particular infimal VAlue theOREM}

In the proof of our subsequent infimal value theorem, we shall use the following minimal value result.

Lemma 1. If $f$ is a continuous function of $\mathbb{R}$ to itself such that

$$
\lim _{t \rightarrow-\infty} f(t)=+\infty \quad \text { and } \quad \lim _{t \rightarrow+\infty} f(t)=+\infty,
$$

then there exists $\tau \in \mathbb{R}$ such that

$$
f(\tau)=\min _{t \in \mathbb{R}} f(t) .
$$

Proof. By using the above limit conditions, we can see that there exists $r>0$ such that

$$
f(0) \leq f(t)
$$

whenever either $t<-r$ or $r<t$. Now, by a basic theorem on continuous functions, we can state that there exists $\tau \in[-r, r]$ such that

$$
f(\tau) \leq f(t)
$$

for all $t \in[-r, r]$. Now, since $f(\tau) \leq f(0)$, it is clear that the above inequality also holds for all $t \in \mathbb{R} \backslash[-r, r]$. 
Our colleague, Mihály Bessenyei has observed that the following infimal value theorem can also be proved in a more elementary way, without using the standard methods of analysis for finding local and global minima. However, our subsequent proof can be used to illustrate these methods in classrooms.

Theorem 5. For any $\alpha, \beta \in \mathbb{R}$, with $\alpha \geq 0$ and $|\beta| \leq 1$, we have

$$
\inf _{t \in \mathbb{R}}\left(\sqrt{t^{2}+\alpha}+\beta t\right)=\sqrt{\alpha\left(1-\beta^{2}\right)} .
$$

Proof. Define

$$
f_{\alpha \beta}(t)=\sqrt{t^{2}+\alpha}+\beta t
$$

for all $t \in \mathbb{R}$. Then, in particular

$$
f_{0 \beta}(t)=\sqrt{t^{2}}+\beta t=|t|+\beta t= \begin{cases}(\beta+1) t & \text { for } t \geq 0, \\ (\beta-1) t & \text { for } t<0 .\end{cases}
$$

Hence, since $\beta+1 \geq 0$ and $\beta-1 \leq 0$, we can see that $f_{0 \beta}(t) \geq 0$ for all $t \in \mathbb{R}$. Now, since $f_{0 \beta}(0)=0$, it is clear that

$$
\min _{t \in \mathbb{R}} f_{0 \beta}(t)=0=\sqrt{0\left(1-\beta^{2}\right)} .
$$

This shows that the required equality is true in the particular case $\alpha=0$. Therefore, in the sequel, we may assume that $\alpha \neq 0$, and thus $\alpha>0$.

Now, we can also note that

$$
f_{0 \beta}(t)=\sqrt{t^{2}}+\beta t<\sqrt{t^{2}+\alpha}+\beta t=f_{\alpha \beta}(t)
$$

for all $t \in \mathbb{R}$. Hence, by the nonnegativity of the function $f_{0 \beta}$, we can see that $f_{\alpha \beta}(t)>0$ for all $t \in \mathbb{R}$. Moreover, we can note that

$$
\begin{aligned}
& \lim _{t \rightarrow-\infty} f_{\alpha 1}(t)=\lim _{t \rightarrow-\infty}\left(\sqrt{t^{2}+\alpha}+t\right) \\
& \quad=\lim _{t \rightarrow-\infty} \frac{\left(\sqrt{t^{2}+\alpha}\right)^{2}-t^{2}}{\sqrt{t^{2}+\alpha}-t}=\lim _{t \rightarrow-\infty} \frac{\alpha}{\sqrt{t^{2}+\alpha}-t}=0
\end{aligned}
$$

and

$$
\begin{aligned}
\lim _{t \rightarrow+\infty} f_{\alpha(-1)}(t) & =\lim _{t \rightarrow+\infty}\left(\sqrt{t^{2}+\alpha}-t\right) \\
& =\lim _{t \rightarrow+\infty} \frac{\left(\sqrt{t^{2}+\alpha}\right)^{2}-t^{2}}{\sqrt{t^{2}+\alpha}+t}=\lim _{t \rightarrow+\infty} \frac{\alpha}{\sqrt{t^{2}+\alpha}+t}=0 .
\end{aligned}
$$

Hence it is clear that

$$
\inf _{t \in \mathbb{R}} f_{\alpha 1}(t)=0=\sqrt{\alpha\left(1-1^{2}\right)} \quad \text { and } \quad \inf _{t \in \mathbb{R}} f_{\alpha(-1)}(t)=0=\sqrt{\alpha\left(1-(-1)^{2}\right)} .
$$


This shows that the required equality is true in the particular case $|\beta|=1$. Therefore, in the sequel, we may assume that $|\beta| \neq 1$, and thus $|\beta|<1$.

Now, since $\beta-1<0$ and $\beta+1>0$, we can also note that

$$
\lim _{t \rightarrow-\infty} f_{0 \beta}(t)=+\infty \quad \text { and } \quad \lim _{t \rightarrow+\infty} f_{0 \beta}(t)=+\infty .
$$

Hence, by using that $f_{0 \beta} \leq f_{\alpha \beta}$, we can infer that

$$
\lim _{t \rightarrow-\infty} f_{\alpha \beta}(t)=+\infty \quad \text { and } \quad \lim _{t \rightarrow+\infty} f_{\alpha \beta}(t)=+\infty
$$

also hold. Moreover, we can note that $f_{\alpha \beta}$ is continuous. Thus, by Lemma 3.1, there exists $\tau \in \mathbb{R}$ such that

$$
f_{\alpha \beta}(\tau)=\min _{t \in \mathbb{R}} f_{\alpha \beta}(t) .
$$

Moreover, since $f_{\alpha \beta}$ is differentiable, we can also state that $f_{\alpha \beta}^{\prime}(\tau)=0$. Hence, by using that

$$
f_{\alpha \beta}^{\prime}(\tau)=\frac{1}{2 \sqrt{\tau^{2}+\alpha}} 2 \tau+\beta=\frac{\tau+\beta \sqrt{\tau^{2}+\alpha}}{\sqrt{\tau^{2}+\alpha}},
$$

we can infer that $\tau+\beta \sqrt{\tau^{2}+\alpha}=0$, and thus

$$
\tau=-\beta \sqrt{\tau^{2}+\alpha} .
$$

This implies that $\operatorname{sgn}(\tau)=-\operatorname{sgn}(\beta)$. Moreover, we can also easily see that $\tau^{2}=\beta^{2}\left(\tau^{2}+\alpha\right)$, whence

$$
\tau^{2}=\frac{\alpha \beta^{2}}{1-\beta^{2}}, \quad \text { and thus } \quad|\tau|=\frac{\sqrt{\alpha}|\beta|}{\sqrt{1-\beta^{2}}} .
$$

Hence, by using that

$$
|\tau|=\operatorname{sgn}(\tau) \tau \quad \text { and } \quad|\beta|=\operatorname{sgn}(\beta) \beta=-\operatorname{sgn}(\tau) \beta,
$$

we can infer that

$$
\operatorname{sgn}(\tau) \tau=-\operatorname{sgn}(\tau) \frac{\sqrt{\alpha} \beta}{\sqrt{1-\beta^{2}}}, \quad \text { and thus } \quad \tau=-\frac{\sqrt{\alpha} \beta}{\sqrt{1-\beta^{2}}} .
$$

Namely, if $\operatorname{sgn}(\tau)=0$, then because of $\operatorname{sgn}(\tau)=-\operatorname{sgn}(\beta)$ we also have $\operatorname{sgn}(\beta)=0$. Therefore, not only $\tau=0$, but also $\beta=0$. Now, to complete the proof, it remains only to note that

$$
\begin{aligned}
f_{\alpha \beta}(\tau)= & \sqrt{\tau^{2}+\alpha}+\beta \tau=\sqrt{\frac{\alpha \beta^{2}}{1-\beta^{2}}+\alpha}-\frac{\sqrt{\alpha} \beta^{2}}{\sqrt{1-\beta^{2}}} \\
& =\frac{\sqrt{\alpha \beta^{2}+\alpha-\alpha \beta^{2}}}{\sqrt{1-\beta^{2}}}-\frac{\sqrt{\alpha} \beta^{2}}{\sqrt{1-\beta^{2}}}=\sqrt{\alpha} \frac{1-\beta^{2}}{\sqrt{1-\beta^{2}}}=\sqrt{\alpha\left(1-\beta^{2}\right)} .
\end{aligned}
$$


Remark 3. Note that if in particular $|\beta| \neq 1$, then we can write minimum instead of infimum in the above theorem.

\section{THE FIRST ILLUSTRATING EXAMPLE}

The following example is a little more difficult than that given in [2].

Example 1. Take

$$
X=\mathbb{R}^{2} \quad \text { and } \quad V=\mathbb{R}(1,1) .
$$

Moreover, define

$$
\varphi(r, r)=r \quad \text { and } \quad p(r, s)=\sqrt{r^{2}+s^{2}}
$$

for all $r, s \in \mathbb{R}$. Then, it is clear that $V$ is a subspace of $X, \varphi$ is a linear function of $V$, and $p$ is a norm on $X$ such that

$$
|\varphi(r, r)|=|r| \leq \sqrt{2}|r|=\sqrt{r^{2}+r^{2}}=p(r, r)
$$

for all $r \in \mathbb{R}$. Thus, in particular, $\varphi$ is dominated by $p$ on $V$.

Moreover, by using Theorem 3.2, we can see that

$$
\begin{array}{r}
q(r, s)=\inf _{u \in \mathbb{R}}(p((r, s)-(u, u))+\varphi(u, u)) \\
=\inf _{u \in \mathbb{R}}(p(r-u, s-u)+\varphi(u, u))=\inf _{u \in \mathbb{R}}\left(\sqrt{(r-u)^{2}+(s-u)^{2}}+u\right) \\
=\inf _{u \in \mathbb{R}} \sqrt{2}\left(\sqrt{\frac{(r-u)^{2}+(s-u)^{2}}{2}}+\frac{u}{\sqrt{2}}\right) \\
=\sqrt{2} \inf _{u \in \mathbb{R}}\left(\sqrt{\frac{(r-u)^{2}+(s-u)^{2}}{2}}+\frac{u}{\sqrt{2}}\right) \\
=\sqrt{2} \inf _{u \in \mathbb{R}}\left(\sqrt{\left(u-\frac{r+s}{2}\right)^{2}+\frac{(r-s)^{2}}{4}}+\frac{1}{\sqrt{2}}\left(u-\frac{r+s}{2}\right)+\frac{r+s}{2 \sqrt{2}}\right) \\
=\sqrt{2}\left(\inf _{u \in \mathbb{R}}\left(\sqrt{\left(u-\frac{r+s}{2}\right)^{2}+\frac{(r-s)^{2}}{4}}+\frac{1}{\sqrt{2}}\left(u-\frac{r+s}{2}\right)\right)+\frac{r+s}{2 \sqrt{2}}\right) \\
=\sqrt{2} \inf _{t \in \mathbb{R}}\left(\sqrt{t^{2}+\frac{(r-s)^{2}}{4}}+\frac{1}{\sqrt{2}} t\right)+\frac{r+s}{2} \\
=\sqrt{2} \sqrt{\frac{(r-s)^{2}}{4}\left(1-\frac{1}{2}\right)+\frac{r+s}{2}}=\frac{|r-s|}{2}+\frac{r+s}{2}=\max \{r, s\}
\end{array}
$$


for all $r, s \in \mathbb{R}$.

Hence, it is clear that, for any $r, s \in \mathbb{R}$, we have

$$
\begin{aligned}
&(r, s) \in W \Longleftrightarrow q(-(r, s))=-q(r, s) \Longleftrightarrow q(-r,-s)=-q(r, s) \\
& \Longleftrightarrow \max \{-r,-s\}=-\max \{r, s\} \Longleftrightarrow-\max \{-r,-s\}=\max \{r, s\} \\
& \Longleftrightarrow \min \{r, s\}=\max \{r, s\} \Longleftrightarrow r=s \Longleftrightarrow(r, s) \in V .
\end{aligned}
$$

Therefore, in the present particular case, $W=V$ holds.

Remark 4. Note that now we have

$$
(r, s)=\frac{s-r}{2}(-1,1)+\frac{r+s}{2}(1,1)
$$

for all $r, s \in \mathbb{R}$.

Therefore, if $f$ is a Hahn-Banach extension of $\varphi$ to $X$, then

$$
\begin{aligned}
f(r, s)= & \frac{s-r}{2} f(-1,1)+\frac{r+s}{2} f(1,1) \\
=\frac{s-r}{2} f(-1,1)+\frac{r+s}{2} \varphi(1,1) & =\frac{s-r}{2} f(-1,1)+\frac{r+s}{2} \\
& =\frac{1-f(-1,1)}{2} r+\frac{1+f(-1,1)}{2} s
\end{aligned}
$$

for all $r, s \in \mathbb{R}$. Moreover, by Theorem 1.7 of [2], we have

$$
-1=-q(1,-1) \leq f(-1,1) \leq q(-1,1)=1 .
$$

Hence, by taking

$$
\lambda=\frac{1-f(-1,1)}{2},
$$

we can see that $\lambda \in[0,1]$ is such that

$$
f(r, s)=\lambda r+(1-\lambda) s
$$

for all $r, s \in \mathbb{R}$. Thus, we can obtain all Hahn-Banach extensions $f$ of $\varphi$ to $X$.

\section{THE SECOND AND THIRD ILLUSTRATING EXAMPLES}

Example 2. Let $X, V$ and $\varphi$ be as in Example 4.1. Moreover, define

$$
p(r, s)=\sqrt{\frac{r^{2}+s^{2}}{2}}
$$

for all $r, s \in \mathbb{R}$. Then, it is clear that $p$ is a norm on $X$ such that

$$
|\varphi(r, r)|=|r|=\sqrt{\frac{r^{2}+r^{2}}{2}}=p(r, r)
$$

for all $r \in \mathbb{R}$. Thus, in particular, $\varphi$ is dominated by $p$ on $V$. 
Moreover, quite similarly as in Example 4.1, we can see that

$$
\begin{gathered}
q(r, s)=\inf _{u \in \mathbb{R}}(p(r-u, s-u)+\varphi(u, u))= \\
=\inf _{u \in \mathbb{R}}\left(\sqrt{\frac{(r-u)^{2}+(s-u)^{2}}{2}}+u\right) \\
=\inf _{u \in \mathbb{R}}\left(\sqrt{\left(u-\frac{r+s}{2}\right)^{2}+\frac{(r-s)^{2}}{4}}+\left(u-\frac{r+s}{2}\right)\right)+\frac{r+s}{2} \\
=\inf _{t \in \mathbb{R}}\left(\sqrt{t^{2}+\frac{(r-s)^{2}}{4}}+t\right)+\frac{r+s}{2} \\
=\sqrt{\frac{(r-s)^{2}}{4}(1-1)}+\frac{r+s}{2}=\frac{r+s}{2}
\end{gathered}
$$

for all $r, s \in \mathbb{R}$.

Hence, it is clear that

$$
q(-(r, s))=q(-r,-s)=\frac{-r-s}{2}=-\frac{r+s}{2}=-q(r, s)
$$

for all $r, s \in \mathbb{R}$, and thus $q$ is an odd function of $X$. Therefore, in the present particular case, $W=X$ holds.

Remark 5. Now, by Theorem 1.1, we can state that $q$ is the unique Hahn-Banach extension of $\varphi$ to $X$.

Example 3. Take

$$
X=\mathbb{R}^{3} \quad \text { and } \quad V=\mathbb{R}(1,1,0) .
$$

Moreover, define

$$
\varphi(r, r, 0)=r \quad \text { and } \quad p(r, s, t)=\sqrt{\frac{r^{2}+s^{2}}{2}}+|t|
$$

for all $r, s, t \in \mathbb{R}$. Then, it is clear that $V$ is a subspace of $X, \varphi$ is a linear function of $V$, and $p$ is a norm on $X$ such that

$$
|\varphi(r, r, 0)|=|r|=\sqrt{\frac{r^{2}+r^{2}}{2}}+|0|=p(r, r, 0)
$$

for all $r \in \mathbb{R}$. Thus, in particular, $\varphi$ is dominated by $p$ on $V$.

Moreover, by making use of the computation given in Example 5.1, we can see that 


$$
\begin{aligned}
q(r, s, t)=\inf _{u \in \mathbb{R}}( & p((r, s, t)-(u, u, 0))+\varphi(u, u, 0)) \\
& =\inf _{u \in \mathbb{R}}(p(r-u, s-u, t)+\varphi(u, u, 0)) \\
= & \inf _{u \in \mathbb{R}}\left(\sqrt{\frac{(r-u)^{2}+(s-u)^{2}}{2}}+|t|+u\right) \\
= & \inf _{u \in \mathbb{R}}\left(\sqrt{\frac{(r-u)^{2}+(s-u)^{2}}{2}}+u\right)+|t|=\frac{r+s}{2}+|t|
\end{aligned}
$$

for all $r, s, t \in \mathbb{R}$.

Hence, it is clear that, for any $r, s, t \in \mathbb{R}$, we have

$$
\begin{aligned}
(r, s, t) \in W \Longleftrightarrow q(-(r, s, t))=-q(r, s, t) & \Longleftrightarrow q(-r,-s,-t)=-q(r, s, t) \\
& \Longleftrightarrow \frac{-r-s}{2}+|-t|=-\left(\frac{r+s}{2}+|t|\right) \\
& \Longleftrightarrow|-t|=-|t| \Longleftrightarrow 2|t|=0 \Longleftrightarrow|t|=0 \Longleftrightarrow t=0 .
\end{aligned}
$$

Therefore, in the present particular case, $W=\mathbb{R} \times \mathbb{R} \times\{0\}$, and thus $V \neq W \neq X$ holds.

Remark 6. Note that now we have

$$
(r, s, t)=t(0,0,1)+(r, s, 0)
$$

for all $r, s, t \in \mathbb{R}$.

Therefore, if $f$ is a Hahn-Banach extension of $\varphi$ to $X$, then by Theorem 1.1 we have

$$
\begin{aligned}
f(r, s, t)=t f(0,0,1)+f(r, s, 0) & =t f(0,0,1)+q(r, s, 0) \\
& =t f(0,0,1)+\frac{r+s}{2}=\frac{1}{2} r+\frac{1}{2} s+f(0,0,1) t
\end{aligned}
$$

for all $r, s, t \in \mathbb{R}$. Moreover, by Theorem 1.7 of [2], we have

$$
-1=-q(0,0,-1) \leq f(0,0,1) \leq q(0,0,1)=1 .
$$

Hence, by taking

$$
\lambda=f(0,0,1),
$$

we can see that $\lambda \in[0,1]$ such that

$$
f(r, s, t)=\frac{1}{2} r+\frac{1}{2} s+\lambda t
$$

for all $r, s, t \in \mathbb{R}$. Thus, we can obtain all Hahn-Banach extensions $f$ of $\varphi$ to $X$. 
Acknowledgement. The authors are indebted to Árpád Száz for transforming their results in the present form.

\section{REFERENCES}

[1] G. Buskes, "The Hahn-Banach theorem surveyed," Dissertationes Math. (Rozprawy Mat.), vol. 327, p. 49, 1993.

[2] T. Glavosits and A. Száz, "The infimal convolution can be used to easily prove the classical HahnBanach theorem," Tech. Rep., Inst. Math., Univ. Debrecen, vol. 12, pp. 1-10, 2009.

[3] J.-J. Moreau, "Inf-convolution, sous-additivité, convexité des fonctions numériques," J. Math. Pures Appl. (9), vol. 49, pp. 109-154, 1970.

[4] L. Narici and E. Beckenstein, "The Hahn-Banach theorem: the life and times," Topology Appl., vol. 77, no. 2, pp. 193-211, 1997.

[5] T. Strömberg, “The operation of infimal convolution," Dissertationes Math. (Rozprawy Mat.), vol. 352 , p. 58, 1996.

Authors' addresses

Tamás Glavosits

University of Debrecen, Institute of Mathematics, Pf. 12, H-4010 Debrecen, Hungary

E-mail address: glavo@math.klte.hu

Csaba Kézi

University of Debrecen, Institute of Mathematics, Pf.

12 , H-4010 Debrecen, Hungary

E-mail address: kezicsaba@math.klte.hu 